\title{
Serum antibodies and jejunal histology in giardiasis associated with malabsorption
}

\author{
MARIAN J. RIDLEY AND D. S. RIDLEY \\ From the Hospital for Tropical Diseases, St. Pancras Way, London NW1 OPE
}

SYNOPSIS An immunofluorescent test, using Giardia lamblia cysts as antigen, gave positive results in 32/36 cases of giardiasis with malabsorption, $0 / 2$ cases of giardiasis without malabsorption, $\subseteq$ and $0 / 17$ control patients without giardiasis or malabsorption. The test was positive in 10/34 patients $\overrightarrow{\dot{\omega}}$ with malabsorption in whom $G$. lamblia could not be detected by stool examination or biopsy; some of these cases were presumed to be cryptic giardiasis. There was a crude correlation between antibody titre and the severity of the histological lesion in the jejunum. The finding of a reliable source of antigen remains a problem.

It is now generally accepted that Giardia lamblia is a potential cause of malabsorption or gastrointestinal symptoms (see Petersen (1972) for a review of this and other aspects of giardiasis). The incidence of infection with this parasite appears to be on the increase and at the same time it is becoming apparent that latent infections occur in which the organism can be demonstrated in duodenal juice or jejunal biopsy though not in faeces (Yardley et al, 1964; Ament and Rubin, 1972; Petersen, 1972; Kamath and Murugasu, 1974). The question arises, therefore, how many cases of malabsorption may be due to latent giardiasis.

As far as we are aware, no work has been reported on the serological response to $G$. lamblia. In this paper the results obtained with an indirect immunofluorescent test (IFT) on the sera of patients with proved or possible giardiasis and malabsorption are evaluated, the object being to determine whether there was any antibody response to this parasite and, if so, whether it was of any diagnostic value. Having demonstrated a serum antibody in a high proportion of cases, we thought that a correlation of the titre with the histological changes in the jejunum might possibly shed some light on the pathogenesis of this form of malabsorption.

\section{Material and Methods}

PATIENTS

Patients became available as a result of studies being conducted at this hospital on malabsorption and

Received for publication 24 June 1975. giardiasis (Tomkins et al, 1974). The majority of $\vec{\oplus}$ cases were overland travellers to Asia, recently returned to this country. Malabsorption was diagnosed by the clinician on the basis of symptomatology, supported by faecal fat excretion, glucose, lactose, and xylose tolerance tests, and Schilling

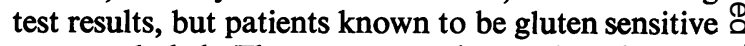
were excluded. The present study was based on $70 \vec{\circ}$ cases of malabsorption together with two patients with asymptomatic giardiasis and 17 non-tropical patients without relevant symptoms who were used as controls.

\section{DETECTION OF G. lamblia}

One or two stool examinations for patients were carried out in all cases using the modified formolether concentration method of Allen and Ridley (1970) on every occasion.

Jejunal juice was aspirated from the upper jejunum within $10 \mathrm{~cm}$ of the ligament of Treitz in 16 of the patients towards the end of the trial. Mucus was of examined for trophozoites or pre-cysts.

\section{JEJUNAL BIOPSY}

Jejunal biopsy was carried out in all cases. Specimens were flattened against a watch glass, or in some cases on filter paper, and fixed in the following fluid: $\mathbb{E}$ formaldehyde $20 \mathrm{ml}$, glacial acetic $3 \mathrm{ml}$, mercuric chloride $2 \mathrm{~g}$, sucrose $1 \mathrm{~g}$, sodium chloride $0.5 \mathrm{~g}$, distilled water to $100 \mathrm{ml}$. After $1 \frac{1}{2}$ to $2 \mathrm{hr}$ the specimen was transferred directly to absolute alcohol, processed, and stained with haematoxylin-eosin. Any specimens that proved to be wrongly orientated in the block were disregarded. More recently, specimens 
have been fixed in some cases in $8 \%$ glutaraldehyde in cacodylate buffer at $\mathrm{pH} 7 \cdot 2$, in which they were left overnight or longer at $4^{\circ} \mathrm{C}$. Examination under the dissecting microscope was carried out by the clinician with the specimen in the fixative.

PAS and reticulin stains were used to demonstrate basement membranes in some specimens. Iron haematoxylin, Mallory's stain, PTAH, and a mixture of toluidine blue and azure blue were used on occasion to demonstrate the parasite. Phosphatases, esterases, and fat were demonstrated in a few specimens by appropriate methods.

\section{ANTIGEN}

G. lamblia antigen for IFT was prepared in three ways:

(1) Trophozoites in duodenal juice were smeared directly on to a slide, or alternatively they were washed in saline and centrifuged. Smears were examined by IFT without delay.

(2) Sections of two jejunal biopsies containing numerous trophozoites in the lumen or crypts were cut at $8 \mu \mathrm{m}$ on the cryostat. The unfixed specimens were snap-frozen and stored at $-70^{\circ} \mathrm{C}$ until required for IFT.

(3) Cysts were collected separately from 10 patients heavily infected with $G$. lamblia and with evidence of malabsorption. Ten gram quantities of stool were mixed with $20 \mathrm{ml} \mathrm{33 \%}$ zinc sulphate and sieved through a wire sieve with $425 \mu \mathrm{m}$ pores. The supernatant was poured into a wide centrifuge tube and centrifuged at $2500 \mathrm{rev} / \mathrm{min}$ for $1 \mathrm{~min}$. The mixture was allowed to stand for 5 min after which the top layer was pipetted off. About $10 \mathrm{ml}$ saline was added to this, then $3 \mathrm{ml}$ ether, and the solution was mixed vigorously using a 'whirlimixer' for 15 $\mathrm{sec}$. The contents were centrifuged at $2500 \mathrm{rev} / \mathrm{min}$ for $1 \mathrm{~min}$, the supernatant was discarded, and the deposit was washed twice with saline and then re-centrifuged. The final deposit consisted of a clean heavy concentration of cysts which was diluted with 1-2 $\mathrm{ml}$ saline. The concentrate was frozen at $-70^{\circ} \mathrm{C}$ for at least $10 \mathrm{~min}$ and thawed, and smears were made. They were dried for 10 min using a hair dryer, fixed in methanol for $10 \mathrm{~min}$, washed in phosphate buffered saline at $\mathrm{pH} 7.4$ for $5 \mathrm{~min}$, and gently blotted immediately before carrying out the IFT. The concentrate could be stored at $-70^{\circ} \mathrm{C}$ until ready for use, but not the smears.

The standard IFT technique using FITC conjugated antihuman globulin was carried out with each of the three types of antigen. Serum was tested at dilutions of $1 / 10,1 / 50$, and $1 / 100$. The microscope was a Leitz ortholux with darkground condenser and interference filter.

\section{Results}

\section{IMMUNOFLUORESCENCE}

Trophozoites in stools made unsatisfactory antigen because of the small numbers and loss in washing. When smeared directly on to the slide they failed to give reproducible results with the IFT, probably because of the adherence of duodenal juice.

Trophozoites in frozen sections were much fewer than in fixed histological preparations. They were too scanty to provide a satisfactory antigen, although those present were observed to fluoresce.

Out of 10 attempts to make a faecal cyst antigen, only two concentrates proved to be satisfactory. These were from stools containing a number of precystic or immature cyst forms, ovoid bodies with a very thin non-refractile cell wall and apparently only one nucleus. In other respects the internal structure was typical of $G$. lamblia. With precystic forms there was cytoplasmic fluorescence, whereas with mature cysts only the cell wall fluoresced.

The IFT results at a screening dilution of $1 / 10$, using the pre-cyst antigen, are shown in the table; $32 / 36$ patients with giardiasis and malabsorption were positive against $0 / 17$ normal controls. In other cases of malabsorption the results were variable. When positive sera were taken to titre it was found that one-third or more reached $1 / 50$ or higher, the highest observed titre being $1 / 160$. There was no significant difference in titre between groups 1 and 2 .

\section{Effect of Treatment}

Sera from 19 serologically positive patients in group 1 were tested one month or more after treatment for giardiasis. The IFT showed a fall in titre in 11 cases and no change in eight. Similarly, one out of two patients in group 2 became negative after treatment. It would be reasonable to expect that more cases would have shown a fall in titre if they could have been re-examined after a longer time interval.

\section{IMMUNOGLOBULINS}

The IFT antibody was tested by the use of conjugated antihuman-globulin sera monospecific for the three main classes of immunoglobulin. In six out of six giardiasis sera the IFT antibody was IgG. Tests with $\operatorname{IgA}$ and $\operatorname{IgM}$ were negative.

Monospecific antihuman globulin was also used in a direct fluorescent test on cryostat sections of jejunums in 10 cases of giardiasis with a short history of about one month's duration and moderately severe histological change (grade II, see below). Plasma cells in the lamina propria showed increased fluorescence with the anti-IgM serum by comparison with a normal control jejunum. Anti-IgA and IgG 


\begin{tabular}{lllll}
\hline Patient Group & \multicolumn{3}{l}{ IFT } \\
\cline { 2 - 4 } \cline { 3 - 4 } & Total & Negative & Positive \\
\hline 1 & $\begin{array}{c}\text { Malabsorption; G. lamblia found } \\
\text { in stool or duodenal juice }\end{array}$ & 36 & 4 & 32 \\
2 & $\begin{array}{c}\text { Malabsorption; G.lamblia not } \\
\text { found }\end{array}$ & 34 & 24 & 10 \\
3 & $\begin{array}{c}\text { G.lamblia in stools; no } \\
\text { malabsorption }\end{array}$ & 2 & 2 & 0 \\
4 & $\begin{array}{c}\text { No G.lamblia nor intestinal } \\
\text { symptoms }\end{array}$ & 17 & 17 & 0 \\
\hline
\end{tabular}

Table IFT results with $\mathrm{G}$. lamblia antigen in malabsorption

sera gave fluorescence similar to the control jejunum. It was concluded that in the early stage of the infection, at least, immunoglobulin production by plasma cells was predominantly IgM.

In an unselected series of giardiasis sera on which immunoglobulins were estimated, the values for IgA, IgG, and IgM were found to be within normal limits. There was no case of IgA deficiency. It was concluded that although immunodeficiency may be associated with giardiasis, the latter is not necessarily the cause of an immunoglobulin deficiency.

Tests for antireticulin and antimitochondrial antibodies using rat liver and kidney and human stomach and thyroid were negative in the sera of all the giardiasis cases, as they were also in sera of tropical sprue. However, a control coeliac disease serum was positive for antireticulin but not antimitochondrial antibody.

\section{HISTOLOGY}

After a trial of many possible modifications and alternatives the mercuric chloride fixative was found to give rapid fixation with excellent preservation of histological detail in the jejunal mucosa. On the other hand, by loosening or dissolving adherent mucus it caused the washing away during processing of many $G$. lamblia trophozoites. The parasites were best seen in glutaraldehyde fixed specimens.

The histological changes of malabsorption in jejunal mucosa were graded in a simple manner as follows:

Grade $O$ (within normal limits)

The architecture of the majority of villi was normal. Epithelium was normal for the most part. The villus/ crypt ratio was about 5:1.

\section{Grade I}

Most villi were finger-shaped but a little broadened due to oedema or a mild plasma cell infiltrate. The epithelium of some villi usually showed a nuclear shift from the basal to the superficial part of the cell; in the basal part there was some vesiculation and often infiltration of a few lymphocytes. Crypts were normal. The villus/crypt ratio was about 4:1.

\section{Grade II}

Most villi were broadened and shortened by a heavy $\cong$ infiltrate, predominantly of plasma cells. A few क might be relatively normal, a few others quite $\overrightarrow{0}$ abnormal or fused. The epithelium, often cuboidal, $\vec{\overrightarrow{ }}$ was usually disorganized by a lymphocytic infiltrate, $\vec{\omega}$ and sometimes there was marked vesiculation also. The crypts were lengthened and the villus/crypt ratio was about $2 \frac{1}{2}: 1$.

\section{Grade III}

Villi were fused or bow-shaped, with a heavy 을 infiltrate in the lamina propr ia and epithelial changes similar to grade II. The villus/crypt ratio was about $1 \frac{1}{2}: 1$.

\section{Grade IV}

The mucosa was almost flat except for some in- $\vec{\varphi}$ dentations, and the crypts were opened on to the surface. The epithelium might be heavily infiltrated and disorganized. The crypts showed numerous mitoses. Although there were no definite villi the total thickness of the mucosa was fairly normal.

Grade $V$ (not seen in giardiasis)

The mucosa was both flat and atrophic.

In the two patients with giardiasis without malabsorption the jejunum was within normal histological limits (grade $\mathrm{O}$ ). In the 36 patients with giardiasis and malabsorption the histological grading was $\mathrm{O}, \mathrm{I}$ or II in four, 12 , and 20 cases respectively. On other occasions the jejunum of patients with giardiasis has been graded III or IV, but there were no such cases in this selies. There was a crude but significant correlation between histological grading and antibody titre (figure).

The 10 patients with malabsorption and a positive IFT, though $G$. lamblia was not found, showed some correlation between titre and grading, with two exceptions that were difficult to explain (grade $\mathbf{O}$ and titre 1/100; and grade IV and titre 1/50). Surprisingly, of 24 patients with malabsorption but neither G. lamblia parasites nor antibody, 14 showed 0 a histologically normal jejunum. The distribution of cases here reflects the exclusion of gluten-sensitive ? forms of enteropathy.

In patients with giardiasis and malabsorption, goblet cells were increased by comparison with gluten-sensitive forms of sprue, and microvilli and the basement membranes of villi and crypts appeared to be better preserved. The chronic inflammatory 


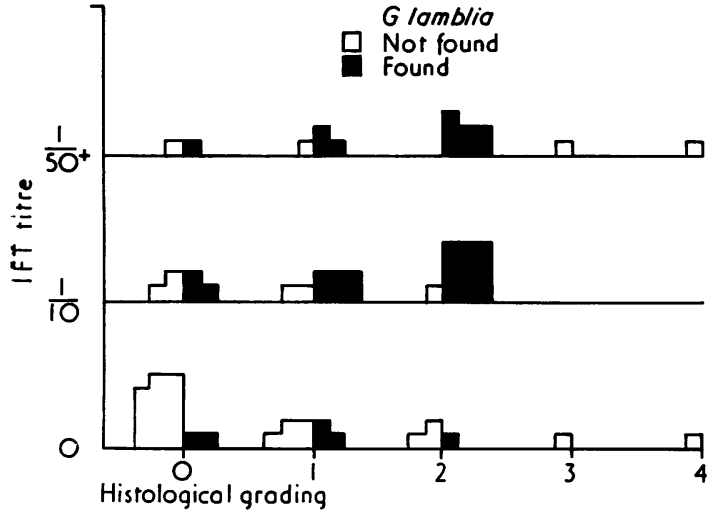

Figure Relationship between IFT titre with G. lamblia antigen and the histological grading of the jejunal lesion in 72 patients in groups 1, 2, and 3.

infiltrate was predominantly plasma cell, but polymorphs were sometimes present. The activity of lymphoid follicles, when they were seen in a section, was parallel to the histological grading. Fat stains did not reveal any abnormality even in grade III biopsies. Acid and alkaline phosphatases and esterases appeared to be normal.

G. lamblia trophozoites in tissue sections proved to be difficult to stain. They were frequently found in the mucus of lumen or crypts, but they were never definitely demonstrated in tissue with any of the stains used or by fluorescence.

\section{OTHER ORGANISMS}

Since Trichomonas vaginalis (another flagellate) is known to be associated with the presence of yeasts in the vagina, and since yeasts are used in culture methods for $G$. lamblia, it was decided to search for yeasts in the centrifuged deposit of jejunal juice of the malabsorption patients. No association with giardiasis was found. Yeasts were detected only in patients treated for tropical sprue with tetracycline.

\section{METHODS OF DETECTING $G$. lamblia}

The present study was unsuitable for an assessment of the relative efficiency of different methods of detecting $G$. lamblia in faeces or intestine. In a parallel study in which jejunal aspirates were examined in all cases, G. lamblia was found in 38; stool concentration and aspirate each produced 35 positives, an efficiency of $92 \%$. When smudge tests (impression smears of biopsies) were included in addition the findings from stool examination represented $85 \%$ of the total (Dr A. M. Tomkins, personal communication). These figures for stools are much higher than those reported by some other workers.

\section{Discussion}

The interest of malabsorption in giardiasis lies in the fact that there is an organism which can reasonably be assumed to be the aetiological agent, but there is doubt how often it invades the tissue. Invasion by the flagellate organisms was demonstrated convincingly by Brandborg et al (1967) in 6/6 cases, and by Morecki and Parker (1967) in one case, but not by other workers. Flagellates are not well adapted for survival in tissue without changing their form, and the discrepant results are hard to account for.

In the present paper a circulating antibody against G. lamblia is demonstrated in $89 \%$ of cases of giardiasis associated with malabsorption, though not in two cases without malabsorption. Whether the antibody is due to invasion of the organisms or merely to absorption of antigen from the lumen of the gut is conjectural, but the rough correlation of antibody titre and the severity of the histological lesion suggest that the antibody might be associated in some way with the pathogenesis of the intestinal lesion. The reason why circulating antibody has not previously been demonstrated may be the difficulty of preparing antigen. Only two stools (both semiliquid, both from children from Africa) containing thin-walled pre-cysts provided suitable material. The cell wall fluorescence observed in mature cysts did not give completely reproducible results.

Despite the clear-cut results with proved positive and proved negative giardiasis, patients with malabsorption in whom G. lamblia was not found were positive in $10 / 34(30 \%)$ cases. This can perhaps be explained by previous observations that $G$. lamblia may not be detected by stool examination even when concentration methods are used; Kamath and Murugasu (1974) detected only 6/12 cases by repeated stool examination, and Ament and Rubin (1972) $3 / 7$, although our figures are higher than these. Thus it may be that serology will prove to be a useful tool for the investigation of patients with malabsorption. But it cannot yet be assumed that all the $10 / 34$ cases were cryptic giardiasis. The possibility in some cases of cross reaction with some antibody against tissue or another enteric organism needs to be excluded. Discrepancies in the correlation between titre and histological grading, and the subsequent clinical follow-up, suggest that two of the cases in this group may have been false positives. There is clearly a need for further work to evaluate these possibilities, which will have to await the finding of a reliable source of antigen. There is some 
hope that culture of the organism, which has so far proved difficult, may become feasible (Meyer, 1970), or that a satisfactory antigen might be obtained by fractionation of cysts.

The only distinct histological difference between the jejunums of giardiasis and coeliac disease is the normal reticulin pattern and smooth basement membranes in the former but not the latter, though there are other differences of degree. One wonders whether the speed with which the jejunum recovers after the successful treatment of giardiasis (Wright et al, 1976) might be connected with the retention of intact basement membranes. It is interesting also that whereas in coeliac disease disruption of the reticulin pattern is associated with antireticulin antibody, in giardiasis there is neither one nor the other.

We are grateful to Dr H. A. K. Rowland and Dr A. M. Tomkins for the collection of biopsy specimens and aspirates and for clinical information, and also for their helpful comments; to $\mathrm{Mr} \mathrm{A}$. $\mathrm{H}$. Moody and Mrs P. L. Hagan for the stool microscopy; and to Dr Deborah Doniach for kindly supplying human stomach and thyroid. We are indebted to the Research Funds of the Hospital for Tropical Diseases for financial support to one of us (M.J.R).

\section{References}

Allen, A. V. H. and Ridley, D. S. (1970). Further observations on the formol-ether concentration technique for faecal parasites. J. clin. Path., 23, 545-546.

Ament, M. E. and Rubin, C. E. (1972). Relation of giardiasis to abnormal intestinal structure and function in gastrointestinal immunodeficiency syndromes. Gastroenterology, 62, 216-226.

Brandborg, L. L., Tankersley, C. B , Gottlieb, S., Barancik, M., and Sartor, V. E. (1967). Histological demonstration of mucosal invasion by Giardia lamblia in man. Gastroenterology, 52, 143-150.

Kamath, K. R., and Murugasu, R. (1974). A comparative study of four methods for detecting Giardia lamblia in children with diarrheal disease and malabsorption. Gastroenterology, 66, 16-21.

Meyer, E. A. (1970). Isolation and axenic cultivation of Giardia trophozoites from the rabbit, chinchilla, and cat. Exp. Parasit., 27, 179-183.

Morecki, R. and Parker, J. G. (1967). Ultrastructural studies of the human Giardia lamblia and subjacent jejunal mucosa in a subject with steatorrhea. Gastroenterology, 52, 151-164.

Petersen, H. (1972). Giardiasis (lambliasis). Scand. J. Gastroent, 7, suppl. 14, pp. 1-44.

Tomkins, A. M., James, W. P. T., Walters, J. H., and Cole, A. C. E. (1974). Malabsorption in overland travellers to India. Brit. med. J., 3, 380-384.

Yardley, J. H., Takano, T., and Hendrix, T. R. (1964). Epithelial and other mucosal lesions of jejunum in giardiasis: jejunal biopsy studies. Bull. Johns Hopk. Hosp., 115, 389-406. 